\title{
Conversion of pre-RISC to holo-RISC by Ago2 during assembly of RNAi complexes
}

\author{
KEVIN KIM, ${ }^{1}$ YOUNG SIK LEE, ${ }^{2}$ and RICHARD W. CARTHEW ${ }^{\mathbf{1}}$ \\ ${ }^{1}$ Department of Biochemistry, Molecular Biology and Cell Biology, Northwestern University, Evanston, Illinois 60208, USA \\ ${ }^{2}$ Division of Biotechnology, College of Life Sciences and Biotechnology, Korea University, Seoul 136-713, South Korea
}

\begin{abstract}
In the Drosophila RNA interference (RNAi) pathway, small interfering RNAs (siRNAs) direct Argonaute2 (Ago2), an endonuclease, within the RNA-induced silencing complex (RISC) to cleave complementary mRNA targets. In vitro studies have shown that, for each siRNA duplex, RISC retains only one strand, the guide, and releases the other, the passenger, to form a holo-RISC complex. Here, we have isolated a new Ago2 mutant allele and provide, for the first time, in vivo evidence that endogenous Ago2 slicer activity is important to mount an RNAi response in Drosophila. We demonstrate in vivo that efficient removal of the passenger strand from RISC requires the cleavage activity of Ago2. We have also identified a new intermediate complex in the RISC assembly pathway, pre-RISC, in which Ago2 is stably bound to double-stranded siRNA.
\end{abstract}

Keywords: RNAi; RISC assembly; Ago2; siRNA; Drosophila

\section{INTRODUCTION}

RNA interference (RNAi) is a post-transcriptional gene silencing process during which endogenous messenger RNA is destroyed upon introduction of the corresponding double-stranded RNA (dsRNA) (Fire et al. 1998). RNAi and other forms of RNA silencing have been observed in a wide variety of organisms such as animals, plants, fungi, and protozoa (Meister and Tuschl 2004).

The initiation of RNAi occurs with the processing of dsRNA into siRNAs (small interfering RNAs), by an RNase III enzyme called Dicer (Meister and Tuschl 2004). In Drosophila, Dicer-2 (Dcr-2) generates these duplex siRNAs. An siRNA then binds to a heterodimer of R2D2 and Dcr-2 proteins to form a R2D2/Dcr-2 initiator complex (RDI) (Liu et al. 2003; Lee et al. 2004; Pham et al. 2004; Tomari et al. 2004a). It has been shown that RDI orients the siRNA duplex so that the $5^{\prime}$ end with lower melting temperature preferentially interacts with Dcr-2 (Tomari et al. 2004b). The strand with the lower melting temperature becomes the single-stranded guide RNA of RISC (RNA-induced silencing complex). RISC uses this single-stranded guide RNA to find

Reprint requests to: Richard W. Carthew, Department of Biochemistry, Molecular Biology and Cell Biology, Northwestern University, 2205 Tech Drive, Evanston, IL 60208, USA; e-mail: r-carthew@northwestern.edu; fax: (847) 467-1380.

Article published online ahead of print. Article and publication date are at http://www.rnajournal.org/cgi/doi/10.1261/rna.283207. complementary mRNA and endonucleolytically cleave target mRNA. Previous studies have identified Ago2 as the catalytic subunit of RISC cleavage activity (Liu et al. 2004; Song et al. 2004). The Piwi domain of Ago 2 is a RNase H-like endonuclease that cleaves a single phosphodiester bond in the target RNA backbone (Liu et al. 2004; Song et al. 2004).

One of the essential steps of RISC formation is dissociation of the paired siRNA strands, and selective retention of one strand (guide) and loss of the other (passenger) from RISC. It has long been thought that double-stranded siRNAs are unwound by an ATP-dependent RNA helicase, activated or recruited with formation of RDI. Consistent with the idea, assembly of RISC in a Drosophila embryo lysate is reduced upon depletion of ATP (Nykanen et al. 2001). Likely candidates have been identified using both genetic and biochemical studies, yet their biochemical roles have not been demonstrated.

Recent biochemical studies have presented a model in which the endonucleolytic activity of Ago2 is responsible for passenger strand dissociation in vitro by means of passenger strand cleavage (Matranga et al. 2005; Rand et al. 2005; Leuschner et al. 2006). In this paper, we used a genetic screen to isolate a new Ago2 allele and demonstrate in vivo that efficient removal of the passenger strand from RISC requires the cleavage activity of Ago2. With this mutant allele, we have also identified a new intermediate complex in the RISC assembly pathway in which Ago2 is stably bound to siRNA before unwinding. 


\section{RESULTS}

\section{Genetic identification of $A g o 2^{V 966 M}$ mutant allele}

We previously performed an EMS-induced mutagenesis screen to identify new and essential components of the RNAi pathway in Drosophila (Lee et al. 2004). Using eye-specific mitotic recombination, we screened animals that had homozygous mutant compound eyes, while all other tissues, including the germ-line, were heterozygous (Stowers and Schwarz 1999; Newsome et al. 2000). Animals with constitutive RNAi against the white gene were examined for variants that had de-repressed white gene activity in the eye (Fig. 1A,B).

Our screen of the third chromosome identified a mutation that partially de-repressed white gene activity (Fig. 1C,D). Animals that were homozygous for the mutation also exhibited white de-repression, and they were both viable and fertile. Complementation analysis with known viable RNAi mutants was performed. The mutation failed to complement a null Ago2 mutation, Ago $2^{414}$ (Fig. 1E,F; Okamura et al. 2004). In addition, a single copy of an Ago2 genomic transgene (Okamura et al. 2004) rescued the
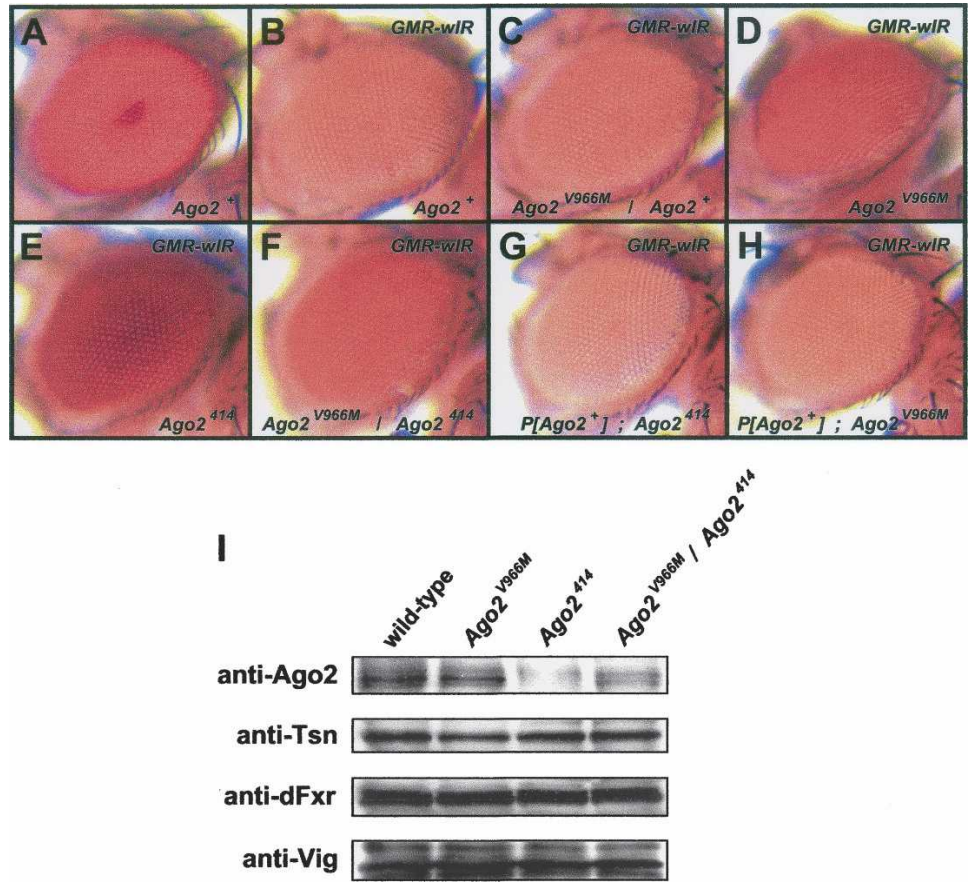

FIGURE 1. Phenotype of the Ago $2^{V 966 M}$ MutantCompound eyes from white ${ }^{+}$adult flies $(A-$

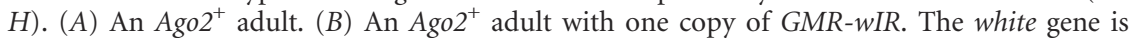
partially silenced, resulting in a pale orange eye color. (C) An adult with one copy of GMR-wIR and heterozygous for Ago2 ${ }^{V 966 M}$. (D) An adult with two copies of GMR-wIR and homozygous for Ago2 ${ }^{V 966 M}$. (E) An adult with two copies of GMR-wIR and homozygous for Ago2 $2^{414}$. (F) An adult with two copies of GMR-wIR and trans-heterozygous for Ago2 $2^{V 966 M}$ and $A g o 2^{414}$. (G) An Ago $2^{414}$ adult homozygote with two copies of GMR-wIR and a single copy of the Ago2 rescue transgene. $(H)$ An Ago $2^{V 966 M}$ adult homozygote with two copies of GMR-wIR and a single copy of the Ago2 rescue transgene. (I) Lysates prepared from wild-type, Ago2 ${ }^{\mathrm{V} 966 \mathrm{M}}, \mathrm{Ago2}^{414}$, and Ago $2^{V 966 M} / A_{g o} 2^{414}$ embryos were separated by SDS-PAGE, blotted onto nitrocellulose membrane, and subjected to Western analysis using antibodies against TSN, VIG, dFXR, and Ago2.
RNAi silencing defect of the mutant chromosome (Fig. $1 \mathrm{G}, \mathrm{H})$. Sequencing of the Ago2 gene from the mutant revealed a base substitution that results in a valine to methionine amino acid substitution in the Piwi domain (Fig. 2B). Based on all of these data, we have identified the mutation as an allele of Ago2 and have named it $A g o 2^{V 966 M}$. The white (RNAi) phenotype of $A g o 2^{V 966 M}$ was not as strong as the Ago2 null phenotype, suggesting that it is not a null mutation. Consistent with this notion, Western blot analysis with anti-Ago2 antibody revealed that there was a normal level of Ago2 protein present in the mutant lies (Fig. 1I)

The Piwi domain of an archaebacterial Ago (Pf-Ago) protein has been shown to resemble the catalytic domain of RNase $\mathrm{H}$, an RNase that cleaves the RNA strand of RNA/ A hybrid duplexes (Song et al. 2004). There are two mo structurally equivalent to two aspartate residues that coordinate a metal ion in RNase H (Yang and Steitz 1995). Critically, the metal ion is at the catalytic core of the RNase $\mathrm{H}$ enzyme. A third coordinating carboxylate varies in its position within the active site of RNase H. Studies of PfAgo suggested that a glutamate in close proximity to the two aspartates was the third coordinating residue (Parker et al. 2004). However, a recent structural study has determined a nearby histidine residue to be the third residue of Pf-Ago (Rivas et al. 2005). Consistent with these structural predictions based on Pf-Ago, mutation of the histidine or either aspartate residue in human Ago2 abolishes the RNA cleavage activity of RISC (Rivas et al. 2005).

The mutation of $A g o 2^{V 966 M}$ changes the GxDV motif into GxDM. This valine residue is highly conserved among all Ago proteins. Modeling of a GxDM variant of the Pf-Ago structure (Song et al. 2004) with PyMol revealed a steric change in the integrity of the structure at the catalytic site (data not shown). We suspect that the V-to-M mutation may alter the metal coordinating properties required for normal catalysis. Recent study of the crystal structure of bacterial Rnase $H$ suggests a two metal ion mechanism (Nowotny et al. 2005). Two metal ions found in the active site of RNase $\mathrm{H}$ are important for activating the nucleophile and stabilizing the transition state. In addition, 
A

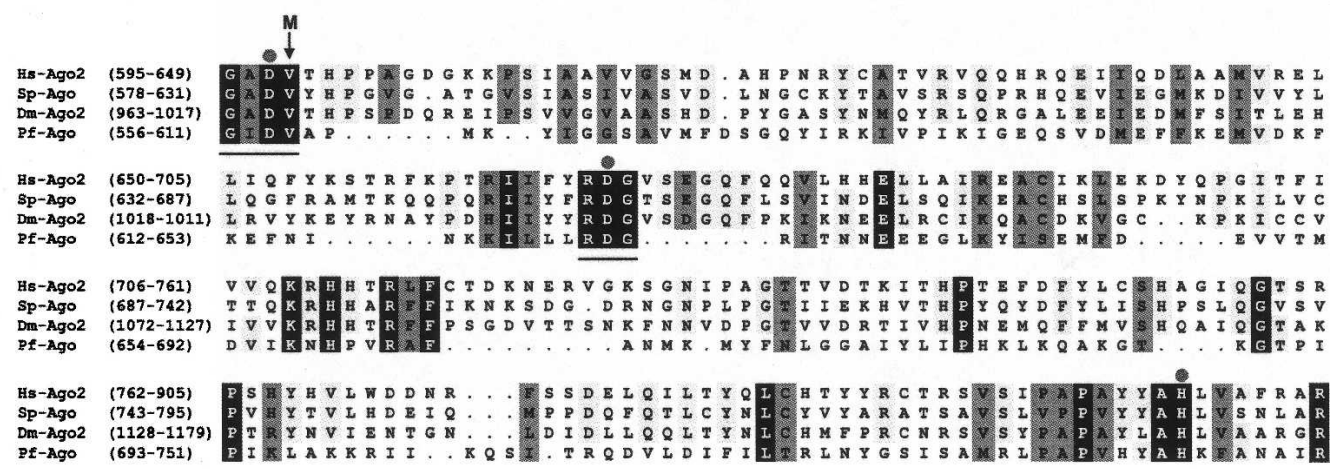

B

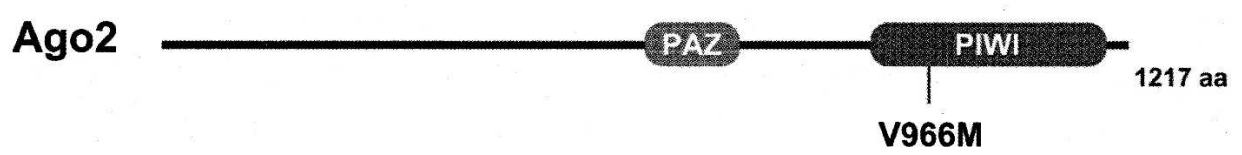

FIGURE 2. Structural characteristics of $A g o 2^{V 966 M}$. (A) Protein sequence alignment of Argonaute Piwi domains of Drosophila (Dm), Human (Hs), Pyrococcus furiosus (Pf). Aligned sequences are color-coded: 100\% conserved residues (black), 75\% conserved residues (light gray), and wellconserved hydrophobic or hydrophilic residues (dark gray). The two conserved motifs, GxDV and RDG, are indicated with a bar below the sequence. Three conserved catalytic residues within the Piwi domain are marked with circles at the top of the alignment. The mutation in Ago2 ${ }^{V 966 M}$ is marked with an arrow above the alignment. (B) Schematic representation of Drosophila Ago2 protein with known domains. The mutation of $A g o 2^{V 966 M}$ is labeled.

efficient catalysis takes place only when the two metal ions are separated by an ideal distance and arranged in appropriate geometric positions (Nowotny et al. 2005). The valine residue affected in $A g o 2^{V 966 M}$ may disrupt the coordination of the metal ion required for RNA cleavage activity. Alternatively, the mutation may disrupt the positioning of target substrate within the catalytic site.

\section{Ago $2^{V 966 \mathrm{M}}$ is defective in holo-RISC formation}

To determine how RNAi is defective in the $\mathrm{Agol}^{\mathrm{V} 966 \mathrm{M}}$ mutant, we performed an in vitro target cleavage assay. Embryo lysates, either from $A g o 2^{V 966 M}$ homozygotes or Ago ${ }^{V 966 M} / A_{g o 2} 2^{414}$ heterozygotes, were greatly impaired in their ability to cleave target mRNAs (Fig. 3A). This suggested that holo-RISC is not functional and/or the mutant is unable to form a structurally intact holo-RISC.

To test if $\mathrm{Ago2}^{\mathrm{V} 966 \mathrm{M}}$ is able to form holo-RISC, we analyzed RISC formation in Ago2 ${ }^{V 966 M}$ mutant embryo lysate by native gel electrophoresis of siRNA-protein complexes. Ago2 ${ }^{\text {V966M }}$ lysate formed a complex closely corresponding to holo-RISC (Fig. 3B). However, the complex was of slower electrophoretic mobility when compared with wild-type holo-RISC (Fig. 3B). Lysate made from $\mathrm{Ago} 2^{\mathrm{V} 966 \mathrm{M}} / \mathrm{Ago} 2^{414}$ embryos showed reduced abundance of the slower complex, indicating that its formation is dependent upon Ago2 (Fig. 3B).

\section{Ago2 ${ }^{V 966 M}$ forms a pre-RISC complex containing duplex siRNA}

We examined RISC formation using a different siRNA, which is highly asymmetric for siRNA strand retention into holo-RISC. This siRNA preferentially retains one strand (top) over the other strand (bottom) due to a G:U wobble at the $5^{\prime}$ end of the guide strand (Fig. 3C). Upon incubation in wild-type lysate, a siRNA containing radiolabeled guide strand formed two discrete low-mobility complexes (Fig. 3C). The slower complex (S) was less abundant than the faster complex (F). Ago2 $2^{V 966 M}$ mutant lysate also formed the two low-mobility complexes, but there were higher levels of the $S$ complex relative to the $F$ complex. When a siRNA containing radiolabeled passenger strand was incubated in wild-type or Ago $2^{V 966 M}$ lysate, only the $S$ complex was observed, with more of this complex observed in the mutant reaction (Fig. 3C). The inability to observe the $\mathrm{F}$ complex with labeled passenger strand indicates that the $\mathrm{F}$ complex selectively associates with guide strand RNA. In contrast, the $S$ complex associates with either strand equivalently. This would suggest that the F complex contains unwound single-stranded siRNA whereas the $\mathrm{S}$ complex contains duplex siRNA.

If our hypothesis is correct about the RNA composition of the two RISC complexes, then we would predict that target mRNA (complementary to the guide siRNA strand) should be able to associate with the $\mathrm{F}$ complex but not the $S$ complex. We performed a RISC formation assay with 


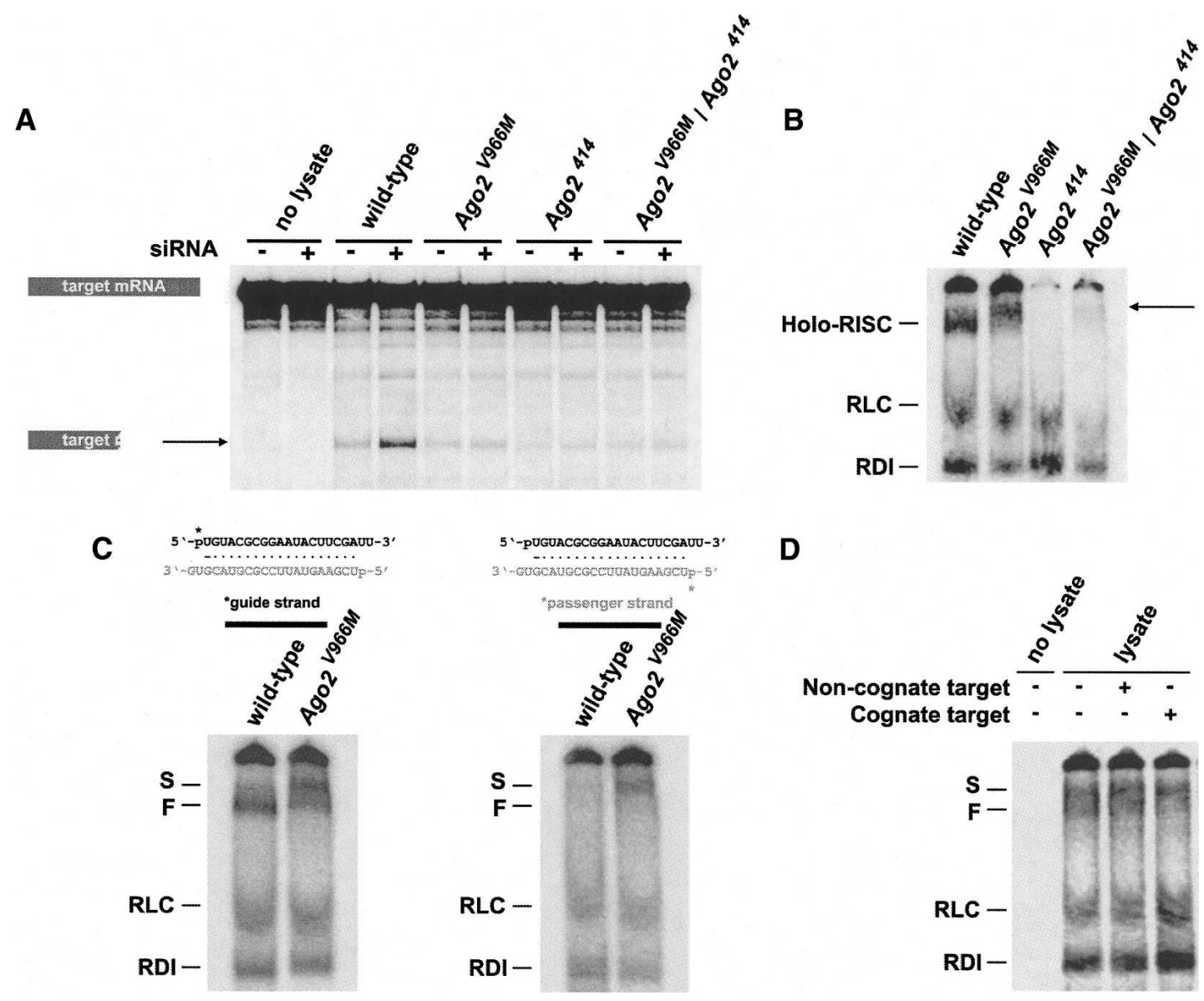

FIGURE 3. Biochemical characterization of $A g o 2^{V 966 M}$. (A) In vitro target RNA cleavage assay. Lysates from wild-type, $A g o 2^{V 966 M}, A g o 2^{414}$, and Ago ${ }^{V 966 M} / A_{g o 2} 2^{414}$ embryos were incubated with $5^{\prime}$-labeled Pp-luc target mRNA and unlabeled cognate siRNA duplex, as indicated. The 5' product from RISC activity is indicated and comigrates on the gel with a cleavage product from siRNA-independent nuclease activity. (B) RISC formation assay. Lysates from wild-type and Ago2 mutant embryos were incubated with labeled $P p$-luc siRNA, and complexes were resolved by native gel electrophoresis. RDI and RLC intermediate complexes are indicated. The complex (arrow) closely corresponding to holo-RISC in Ago $2^{V 966 M}$ mutant was of slower electrophoretic mobility when compared with wild-type holo-RISC. Ago2 $2^{V 966 M} / A_{g o 2} 2^{414}$ embryo lysate showed reduced abundance of the slower complex. (C) RISC formation with a highly asymmetric siRNA. Either the guide (top) or the passenger (bottom) strand was $5^{\prime}$-radiolabeled. Both wild-type and $A g o 2^{V 966 M}$ lysates form two discrete low-mobility complexes when incubated with radiolabeled guide strand. In wild-type lysate, the slower complex (S) was less abundant than the faster complex (F). In Ago2 ${ }^{V 966 M}$ lysate, the relative amount of $\mathrm{S}$ complex increased while F complex decreased. When incubated with radiolabeled passenger strand, both wild-type and Ago2 ${ }^{\mathrm{V} 966 \mathrm{M}}$ lysates formed only the $\mathrm{S}$ complex due to asymmetric loading of unlabeled guide strand into holo-RISC. Note that more of the $\mathrm{S}$ complex was formed in $A g o 2^{V 966 M}$ lysate. $(D)$ Radiolabeled guide siRNA strand was incubated with either unlabeled cognate or noncognate $2^{\prime}$-O-methyl target mRNA in wild-type lysate. Addition of cognate target mRNA resulted in the depletion of the F complex, whereas the $S$ complex was not affected. Addition of noncognate mRNA had no effect on either complex.

guide-labeled siRNA and added an unlabeled 2'-Omethylated target mRNA. Addition of cognate target mRNA resulted in depletion of the $\mathrm{F}$ complex, presumably due to a shift in complex mobility, whereas the $\mathrm{S}$ complex was unchanged (Fig. 3D). Addition of noncognate mRNA had no effect on either complex. This result indicates that the $\mathrm{F}$ complex has the potential to specifically associate with a target mRNA, which is a property of holo-RISC.

To directly visualize the nature of the siRNAs in the two complexes, we performed a two-dimensional gel electrophoresis experiment (Fig. 4A). A RISC formation reaction was performed with guide-labeled siRNA. After resolving the complexes in the first dimension by native gel electrophoresis, we excised the entire lane and placed it horizontally on a SDS-polyacrylamide gel. Labeled siRNAs from complexes were then subjected to electrophoresis in the second dimension. We predicted that ribonucleoprotein complexes resolved in the first dimension would dissociate in the second dimension due to SDS-dependent denaturation. This would permit siRNAs to electrophorese as free species. However, SDS treatment in the second dimension did not denature siRNA duplexes, which were easily distinguishable from single-stranded siRNA (Fig. 4B). Thus, we were able to 
A

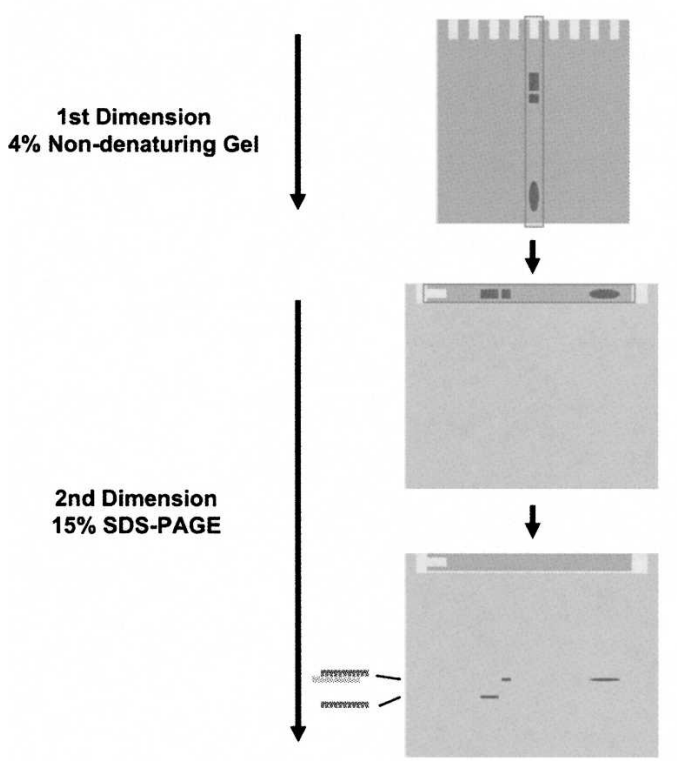

B

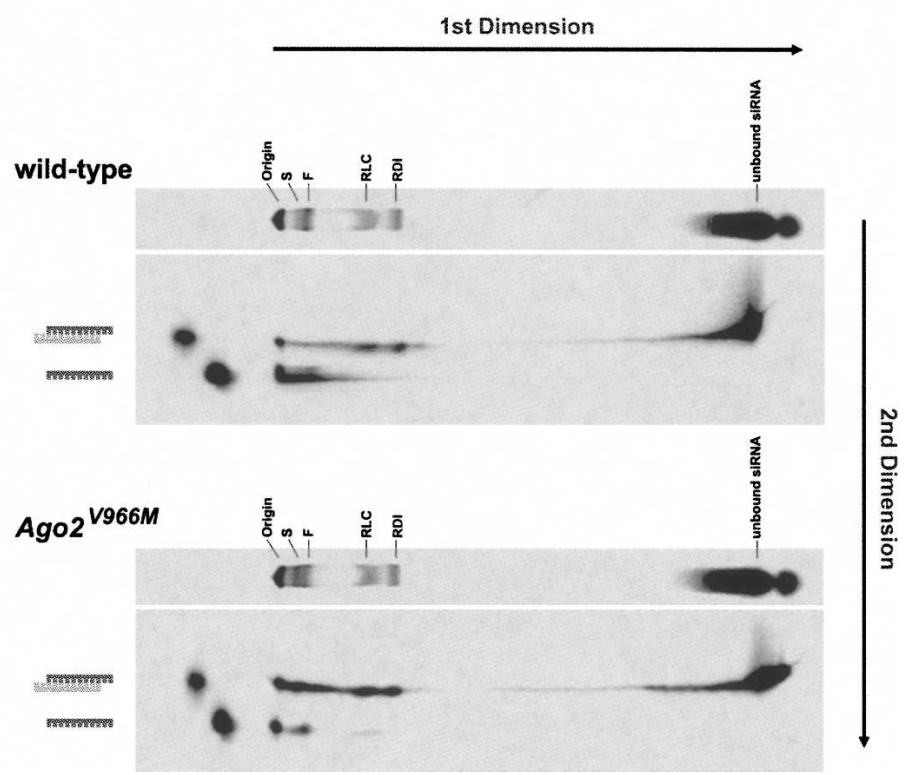

FIGURE 4. $A g o 2^{V 966 M}$ RISC complexes contain primarily duplex siRNA. (A) Schematic representation of two-dimensional gel electrophoresis. RISC formation reaction was performed with guide-labeled siRNA duplex. The complexes were resolved in the first dimension by native gel electrophoresis. The entire lane was than excised and placed horizontally on a 15\% SDS-polyacrylamide gel. Labeled siRNAs from complexes were then subjected to electrophoresis in the second dimension. (B) Both wild-type and $A g o 2^{V 966 M}$ lysates show RDI and RLC complexes that contain duplex siRNA. The low-mobility complexes observed in wild-type lysate primarily contain single-stranded siRNA while those formed in Ago2 ${ }^{V 966 M}$ lysate primarily contain duplex siRNA.

visualize the conformation of siRNAs associated with various complexes formed in the RISC reaction.

When wild-type lysate was subjected to two-dimensional analysis, we observed that both RDI and RLC (RISC loading complex) complexes contained duplex siRNA (Fig. 4B), which is consistent with previous observations (Pham et al. 2004). The $S$ and $F$ complexes contained single-stranded siRNA though some duplex siRNA was also detected (Fig. 4B). However, when $\mathrm{Ago2} 2^{\mathrm{V} 966 \mathrm{M}}$ lysate was analyzed, the low-mobility complexes predominantly contained duplex siRNA with a peak of single-stranded siRNA detected in the F complex (Fig. 4B). Thus, Ago2 stimulates conversion of duplex siRNA into single-stranded siRNA within RISC complexes.

\section{Ago2 ${ }^{\text {V966M }}$ is defective for unwinding of siRNAs and passenger strand cleavage}

Based on all of our observations, it is likely that the $\mathrm{F}$ complex corresponds to holo-RISC. Several lines of evidence are offered in support. First, formation of the $\mathrm{F}$ complex requires Ago2, a key subunit of holo-RISC. Second, both holo-RISC and the F complex contain single-stranded siRNA, and both preferentially retain the guide strand of a siRNA duplex. Finally, holo-RISC and the F complex are able to specifically bind to target mRNA substrate. Our native gel analysis has also identified a distinct Ago2-dependent complex, the $\mathrm{S}$ complex. The formation of the $\mathrm{S}$ complex requires the presence of Ago2 and is more pronounced with deficient catalytic activity of Ago2, which is in contrast to holo-RISC. The S complex appears to contain siRNA in duplex form, and it is unable to bind to target mRNA substrate.

A simple model is that the $\mathrm{S}$ complex is a holo-RISC precursor that contains duplex siRNA, and siRNA unwinding accompanies the conversion of S complex to holo-RISC. If this model is correct, then siRNA unwinding should correlate with complex conversion. Therefore, we determined whether Ago ${ }^{V 966 M}$ mutant lysate shows defective siRNA-unwinding activity comparable to its defective holo-RISC-forming activity. When we examined the rate of single-stranded siRNA production, Ago ${ }^{V 966 M}$ mutant lysate showed a twofold reduced rate of siRNA unwinding (Fig. 5A). A null Ago2 mutant did not produce any single-stranded siRNAs. The strength of the Ago2 ${ }^{V 966 M}$ mutant defect is comparable to the relative changes in $\mathrm{S}$ and $\mathrm{F}$ complex abundance in the mutant (Fig. 3C). Taken together, our data most strongly support a model in which the $\mathrm{S}$ complex is a holo-RISC precursor. Thus, we have renamed it pre-RISC.

Recent in vitro studies have shown that unwinding of siRNA duplex is accompanied by passenger strand cleavage to form holo-RISC (Matranga et al. 2005; Rand et al. 2005; Leuschner et al. 2006). Since Ago2 $2^{V 966 M}$ shows defective siRNA unwinding activity, it was possible that passenger 
A
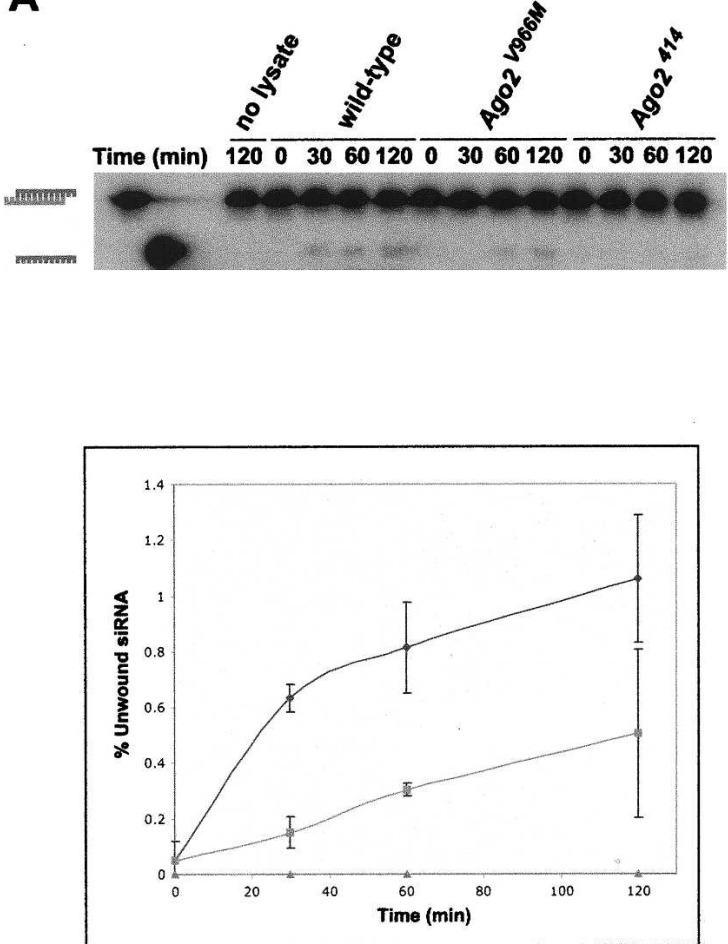

B
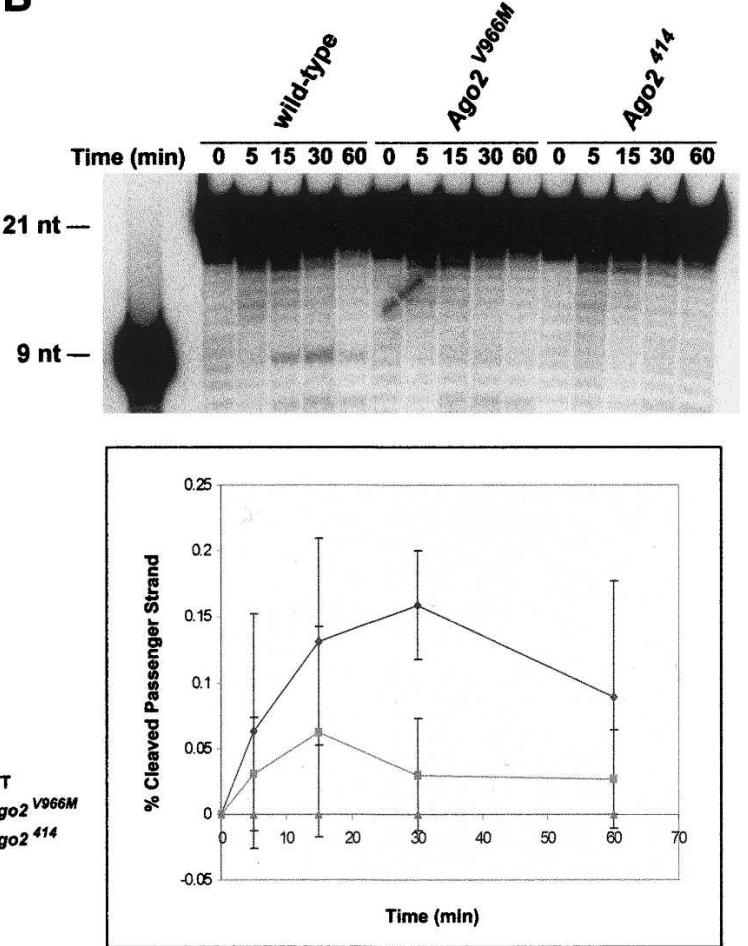

FIGURE 5. $A g o 2^{V 966 M}$ is defective in siRNA duplex dissociation. (A) Guide-labeled siRNA duplex was incubated in wild-type, $A g o 2^{V 966 M}$, or Ago $2^{414}$ lysate. Reactions were resolved on a 15\% SDS-polyacrylamide gel and radioactive signal was quantified by PhosphorImager. The percentage of single-stranded siRNA generated from siRNA duplex was plotted against reaction time. $(B)$ Passenger-labeled siRNA duplex was incubated in the same lysates as $A$. Reactions at various time points were removed, deproteinized, and electrophoresed to visualize passenger strand cleavage products. The 9-nt $5^{\prime}$ cleavage product accumulated early in the reaction and disappeared. Ago $2^{V 966 M}$ mutant lysate produced little passenger strand cleavage product. The percentage of the 9-nt product cleaved from labeled passenger strand was plotted against reaction time.

strand cleavage is also inhibited. To test if $A g o 2^{V 966 M}$ is able to convert passenger strand siRNAs into 9-nucleotide (nt) cleavage products, we performed an in vitro passenger strand cleavage assay. Cleavage of 5 '-labeled passenger strand by wild-type lysate yielded a 9-nt labeled product (Fig. 5B). However, in the Ago $2^{V 966 M}$ mutant lysate, the rate of passenger strand cleavage was strongly reduced (Fig. 5B). These data further support a model where siRNAs are initially loaded as duplexes into Ago2-containing pre-RISC. The catalytic activity of Ago2 is then required to cleave the passenger strand, stimulating it to dissociate from the guide strand and form holo-RISC.

\section{DISCUSSION}

We have isolated a mutation in Drosophila Ago2 that specifically affects its ability to cleave target RNA when the target is associated with holo-RISC. The mutation changes a highly conserved amino acid residue that neighbors one of the nucleophile-coordinating residues within the Piwi domain. Structural modeling predicts that the change disrupts precise positioning of the nucleophile within the catalytic site. Experimentally, this change results in an eightfold reduction in target cleavage activity by holoRISC. The experimental observations are consistent with the structural predictions and provide further evidence that Ago2 has RNA slicer activity in vitro. Moreover, it provides, for the first time, in vivo evidence that endogenous Ago2 slicer activity is important to mount an RNAi response in Drosophila.

The assembly of RISC in vitro follows a pathway of siRNA-protein complexes from RDI to RLC to holo-RISC (Fig. 6). A previous model proposed RLC as the direct precursor to holo-RISC, which harbors both Ago2 and single-stranded guide siRNA (Pham et al. 2004; Tomari et al. 2004a; Pham and Sontheimer 2005). In the present study we have identified a new Ago2-dependent complex, pre-RISC. The difficulty of visualizing pre-RISC in Drosophila embryo extract explains why this complex was not described earlier. One reason for this difficulty requires an understanding of the differences between pre-RISC and holo-RISC. The pre-RISC complex contains duplex siRNA whereas holoRISC contains just the guide strand of the siRNA, the passenger strand having dissociated from holo-RISC. Thus, a key difference between pre-RISC and holo-RISC is the conformation of the associated siRNA. 


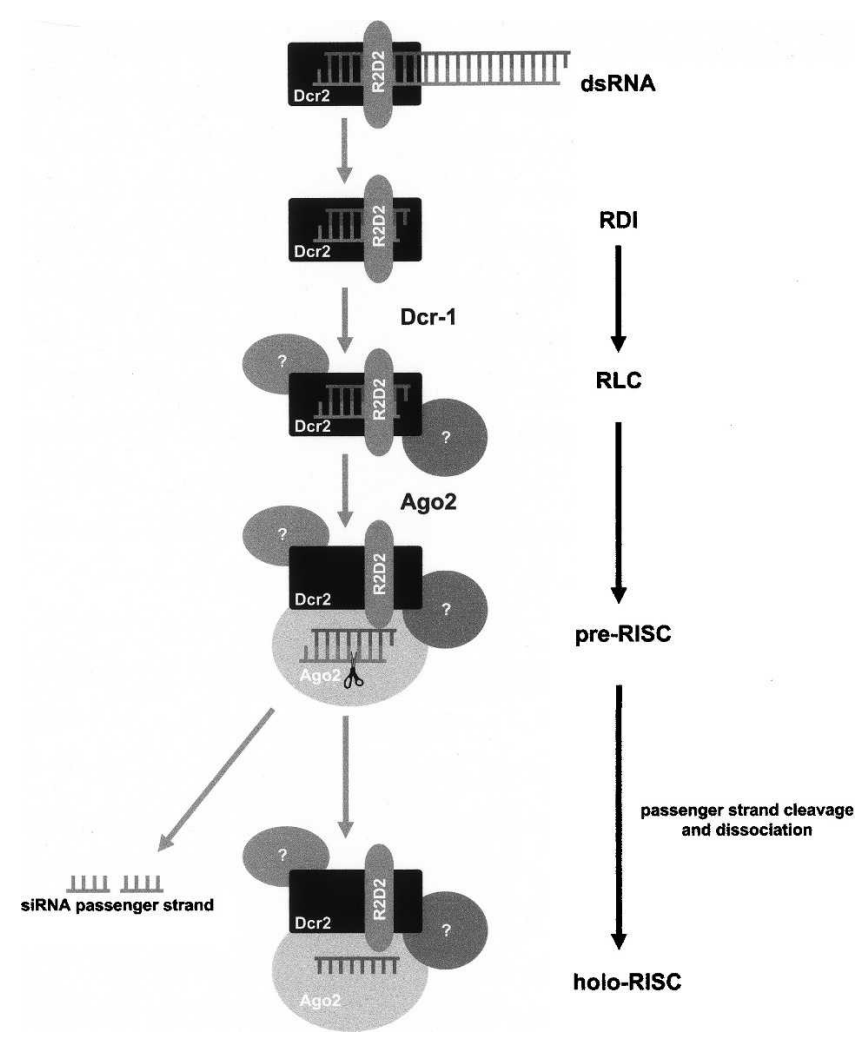

FIGURE 6. Model for RISC assembly in Drosophila in vitro. The siRNA is bound to R2D2/Dcr2 heterodimer to form a RDI complex. The RDI complex then enters a RISC-loading complex (RLC), associating with other RNAi components. RLC then recruits Ago2 to form a pre-RISC complex that contains duplex siRNA. Endonucleolytic activity of Ago2 within the Piwi domain promotes release of the passenger strand from pre-RISC to form holo-RISC. Holo-RISC can then base-pair to complementary single-stranded mRNA substrates for cleavage.

Two simple models could explain the relationship between pre-RISC and holo-RISC. Pre-RISC could be an abortive reaction product in the holo-RISC assembly reaction, formed independently of holo-RISC itself. Alternatively, pre-RISC could be an intermediate complex in the assembly reaction, and act as a holo-RISC precursor. Pulsechase experiments to discern between these models are not possible given our inability to specifically pulse-label preRISC. However, the Ago $2^{V 966 M}$ mutant provides evidence that pre-RISC is a holo-RISC precursor. Mutant extract shows a threefold increase in pre-RISC abundance and twofold decrease in holo-RISC abundance. Furthermore, siRNA strand unwinding is impaired twofold. The most parsimonious explanation for these results is that pre-RISC converts into holo-RISC by siRNA strand dissociation, and the Ago2 mutant is partially impaired for this conversion, resulting in a build-up of the precursor. Our results suggest a revised model in the RISC assembly pathway (Fig. 6).

Recent in vitro studies have shown that unwinding of siRNA duplex is accompanied by cleavage of the ejected
siRNA strand during holo-RISC formation (Matranga et al. 2005; Rand et al. 2005). In the Ago2 $2^{V 966 M}$ mutant, the rate of siRNA strand cleavage and unwinding is reduced. These data support a model where siRNAs are initially loaded as duplexes into Ago2-containing pre-RISC. The catalytic activity of Ago2 is then required to cleave one strand of the duplex, stimulating it to dissociate from the uncleaved strand and ultimately dissociate from RISC. The end result is a RISC retaining one siRNA strand, fully competent to down-regulate transcript targets.

If Ago2-dependent catalysis is obligatory for complex conversion, then we might have expected a more profound assembly block in the $A g o 2^{V 966 M}$ mutant than the effect actually observed. One explanation is that conversion of pre-RISC to holo-RISC is promoted by several factors including Ago2, with each factor augmenting strand dissociation. For example, it is conceivable that Ago2dependent siRNA cleavage leads to some dissociation by passive mechanisms such as temperature whereas other factors such as helicases generate unwinding by other means. Alternatively, strand dissociation might be wholly directed by helicase factors, and strand-cleavage by Ago 2 prevents stable reassociation of the strands. The identity of such a putative helicase remains unknown. The RNA helicase Armitage is required for RISC assembly (Tomari et al. 2004a) but seems to act in the RLC to pre-RISC step of the pathway before unwinding has occurred (data not shown).

\section{MATERIALS AND METHODS}

\section{Fly genetics}

The $A g o 2^{V 966 M}$ mutant was isolated from a genetic screen as described (Lee et al. 2004). The GMR-wIR transgene has been described (Lee and Carthew 2003). Mosaic analysis of the adult eye was performed as described (Lee et al. 2004). Ago $2^{414}$ null and $\mathrm{Ago}^{+}$rescue flies were gifts from Mikiko Siomi (Okamura et al. 2004). Complementation analysis was performed by crossing Ago $2^{V 966 M}$ mutant flies to Ago2 $2^{414}$ mutant flies in GMR-wIR background.

\section{Biochemical methods}

Embryo lysate preparation and target RNA cleavage assay were performed as described (Pham et al. 2004). RISC assembly was assayed by native gel electrophoresis as described (Pham and Sontheimer, 2005). Passenger cleavage reactions were performed as described (Matranga et al. 2005).

\section{Target binding assay}

We used a cognate $P$-luc 2'-O-methyl RNA analog with the sequence $5^{\prime}$-AUCAGUACGGCGGAAUACUUCGAdAdA- $3^{\prime}$ and a noncognate 2'-O-methyl RNA with the sequence $5^{\prime}$-AUCAAGGAAAGUCAUAUGUUAUGGdAdA- $3^{\prime}$. RISC assembly reactions 
were performed with ${ }^{32} \mathrm{P}$-labeled $P p$-luc siRNA duplex for $15 \mathrm{~min}$ in $25^{\circ} \mathrm{C}$. After addition of $1 \mu \mathrm{M}$ cognate or noncognate $2^{\prime}-O$ methyl RNA, reactions were further incubated at $25^{\circ} \mathrm{C}$ for additional $45 \mathrm{~min}$ and then analyzed on a $4 \%$ (40:1) native polyacrylamide gel as described (Pham and Sontheimer 2005).

\section{Two-dimensional gel electrophoresis}

RISC was assembled by adding ${ }^{32} \mathrm{P}$-labeled siRNAs to embryo lysates and resolved by native electrophoresis as described (Pham and Sontheimer 2005) in the first dimension. The entire lane was excised and placed horizontally over 15\% SDS-polyacrylamide gel. This was then subjected to electrophoresis in the second dimension at 10 watts at $4^{\circ} \mathrm{C}$.

\section{Unwinding assay}

${ }^{32} \mathrm{P}$-labeled siRNA duplexes were added to the RISC assembly assay reactions as described above and incubated at $25^{\circ} \mathrm{C}$. Reactions were quenched with $1 \mu \mathrm{L}$ of a heparin mix (Pham et al. 2004) and loaded onto 15\% SDS-polyacrylamide gel and then subjected to electrophoresis at 10 watts at $4^{\circ} \mathrm{C}$.

\section{ACKNOWLEDGMENTS}

We thank Erik Sontheimer, John Pham, and both Sontheimer and Carthew lab members for reagents and helpful discussions. AntiAgo2, anti-Vig, anti-Tsn, and anti-FXR antibodies were generously provided by Greg Hannon. Ago $2^{414}$ null and Ago2 rescue flies were kindly provided by Mikiko Siomi. This work was supported by the NIH.

Received August 23, 2006; accepted September 26, 2006.

\section{REFERENCES}

Fire, A., Xu, S., Montgomery, M.K., Kostas, S.A., Driver, S.E., and Mello, C.C. 1998. Potent and specific genetic interference by double-stranded RNA in Caenorhabditis elegans. Nature 391: 806-811.

Lee, Y.S. and Carthew, R.W. 2003. Making a better RNAi vector for Drosophila: Use of intron spacers. Methods 30: 322-329.

Lee, Y.S., Nakahara, K., Pham, J.W., Kim, K., He, Z., Sontheimer, E.J., and Carthew, R.W. 2004. Distinct roles for Drosophila Dicer-1 and Dicer-2 in the siRNA/miRNA silencing pathways. Cell 117: 69-81.

Leuschner, P.J., Ameres, S.L., Kueng, S., and Martinez, J. 2006. Cleavage of the siRNA passenger strand during RISC assembly in human cells. EMBO Rep. 7: 314-320.
Liu, Q., Rand, T.A., Kalidas, S., Du, F., Kim, H.E., Smith, D.P., and Wang, X. 2003. R2D2, a bridge between the initiation and effector steps of the Drosophila RNAi pathway. Science 301: 1921-1925.

Liu, J., Carmell, M.A., Rivas, F.V., Marsden, C.G., Thomson, J.M., Song, J.J., Hammond, S.M., Joshua-Tor, L., and Hannon, G.J. 2004. Argonaute 2 is the catalytic engine of mammalian RNAi. Science 305: 1437-1441.

Matranga, C., Tomari, Y., Shin, C., Bartel, D.P., and Zamore, P.D. 2005. Passenger-strand cleavage facilitates assembly of siRNA into Ago2-containing RNAi enzyme complexes. Cell 123: 607-620.

Meister, G. and Tuschl, T. 2004. Mechanisms of gene silencing by double-stranded RNA. Nature 431: 343-349.

Newsome, T.P., Asling, B., and Dickson, B.J. 2000. Analysis of Drosophila photoreceptor axon guidance in eye-specific mosaics. Development 127: 851-860.

Nowotny, M., Gaidamakov, S.A., Crouch, R.J., and Yang, W. 2005. Crystal structures of RNase $\mathrm{H}$ bound to an RNA/DNA hybrid: Substrate specificity and metal-dependent catalysis. Cell 121: 1005-1016.

Nykanen, A., Haley, B., and Zamore, P.D. 2001. ATP requirements and small interfering RNA structure in the RNA interference pathway. Cell 107: 309-321.

Okamura, K., Ishizuka, A., Siomi, H., and Siomi, M.C. 2004. Distinct roles for Argonaute proteins in small RNA-directed RNA cleavage pathways. Genes \& Dev. 18: 1655-1666.

Parker, J.S., Roe, S.M., and Barford, D. 2004. Crystal structure of a PIWI protein suggests mechanisms for siRNA recognition and slicer activity. EMBO J. 23: 4727-4737.

Pham, J.W. and Sontheimer, E.J. 2005. Molecular requirements for RNA-induced silencing complex assembly in the Drosophila RNA interference pathway. J. Biol. Chem. 280: 39278-39283.

Pham, J.W., Pellino, J.L., Lee, Y.S., Carthew, R.W., and Sontheimer, E.J. 2004. A Dicer-2-dependent 80 s complex cleaves targeted mRNAs during RNAi in Drosophila. Cell 117: 83-94.

Rand, T.A., Petersen, S., Du, F., and Wang, X. 2005. Argonaute2 cleaves the anti-guide strand of siRNA during RISC activation. Cell 123: 621-629.

Rivas, F.V., Tolia, N.H., Song, J.J., Aragon, J.P., Liu, J., Hannon, G.J., and Joshua-Tor, L. 2005. Purified Argonaute2 and an siRNA form recombinant human RISC. Nat. Struct. Mol. Biol. 12: 340-349.

Song, J.J., Smith, S.K., Hannon, G.J., and Joshua-Tor, L. 2004. Crystal structure of Argonaute and its implications for RISC slicer activity. Science 305: 1434-1437.

Stowers, R.S. and Schwarz, T.L. 1999. A genetic method for generating Drosophila eyes composed exclusively of mitotic clones of a single genotype. Genetics 152: 1631-1639.

Tomari, Y., Du, T., Haley, B., Schwarz, D.S., Bennett, R., Cook, H.A., Koppetsch, B.S., Theurkauf, W.E., and Zamore, P.D. 2004a. RISC assembly defects in the Drosophila RNAi mutant armitage. Cell 116: $831-841$.

Tomari, Y., Matranga, C., Haley, B., Martinez, N., and Zamore, P.D. 2004b. A protein sensor for siRNA asymmetry. Science 306: 13771380 .

Yang, W. and Steitz, T.A. 1995. Recombining the structures of HIV integrase, RuvC and RNase H. Structure 3: 131-134. 

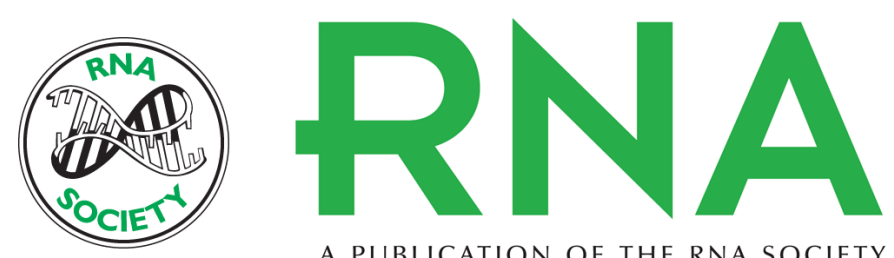

A PUBLICATION OF THE RNA SOCIETY

\section{Conversion of pre-RISC to holo-RISC by Ago2 during assembly of RNAi complexes}

Kevin Kim, Young Sik Lee and Richard W. Carthew

RNA 2007 13: 22-29 originally published online November 22, 2006

Access the most recent version at doi:10.1261/rna.283207

$\begin{array}{ll}\text { References } & \begin{array}{l}\text { This article cites } 22 \text { articles, } 9 \text { of which can be accessed free at: } \\ \text { http://rnajournal.cshlp.org/content/13/1/22.full.html\#ref-list-1 }\end{array}\end{array}$

License

Email Alerting Receive free email alerts when new articles cite this article - sign up in the box at the Service top right corner of the article or click here.

To subscribe to RNA go to:

http://rnajournal.cshlp.org/subscriptions 